\title{
Thrombolysis of stroke mimics via telestroke
}

Vivien H Lee (D), Ravyn Howell, Randheer Yadav, Sharon Heaton, Karen L Wiles, Sushil Lakhani

To cite: Lee VH, Howell R, Yadav $\mathrm{R}$, et al. Thrombolysis of stroke mimics via telestroke. Stroke \& Vascular Neurology 2022;0. doi:10.1136/svn-2020000776

Received 2 December 2020 Accepted 22 December 2021

Check for updates

(C) Author(s) (or their employer(s)) 2022. Re-use permitted under CC BY-NC. No commercial re-use. See rights and permissions. Published by BMJ.

Neurology, The Ohio State University Wexner Medical Center, Columbus, Ohio, USA

Correspondence to Dr Vivien H Lee; vivien.lee@osumc.edu

\section{ABSTRACT}

Background The rate of intravenous tissue plasminogen activator (IVtPA) administered to stroke mimics (SM) occurs in $24 \%-44 \%$ of telestroke series.

Methods We reviewed 270 suspected acute ischaemic stroke (AIS) patients who were evaluated by telestroke and received IVtPA from 1 July 2016 to 30 September 2017 at our academic comprehensive stroke centre.

Results Among 270 AIS patients who received IVtPA via telestroke, 64 (23.7\%) were diagnosed with SM. Compared with patients who had a stroke, the SM group was younger (mean age 56.4 vs $68.2, p<0.0001$ ), more likely to be female (60.9\% vs $45.6 \%, p=0.0324$ ) and had longer door-to-needle times ( 85.3 vs $69.9, p=0.0008$ ). The most common SM diagnoses were migraine 26 (40.6\%), conversion disorder 12 (18.8\%), encephalopathy 7 (10.9\%) and unmasking (9.4\%). Among the SM, migraine and conversion disorder were younger compared with the other subgroups $(p<0.001)$. Functional exam elements were noted more frequently in conversion disorder (66.7\%) and migraine (34.6\%), but rare in other diagnoses $(p=0.006)$. Among the SM, $23(35.9 \%)$ had a history of a prior similar episodes, and $15(23.4 \%)$ had a history of more than 5 spells.

Conclusions In our telestroke programme, $23.7 \%$ of those administered thrombolysis had a final diagnosis of SM.

\section{INTRODUCTION}

The implementation of telestroke has increased the use of intravenous tissue plasminogen activator (IVtPA) at community hospitals by allowing access to stroke expertise remotely and has gained increasingly widespread acceptance. The rate of stroke mimics (SM) in telestroke cohorts is reported to be $24 \%-44 \%$ in large series, although the rate at which SM subsequently go on to receive thrombolysis is not well described in to describe the frequency and characteristics of SM evaluated by telestroke.

\section{METHODS}

We performed a retrospective review of suspected acute ischaemic stroke (AIS) patients who were evaluated by telestroke and received IVtPA from 1 July 2016 to 30 September 2017. Our academic comprehensive stroke programme includes 26 telestroke emergency rooms (ER) throughout Ohio. telestroke cohorts. ${ }^{1}$ The aim of this study was
Our telestroke platform does not allow access to the electronic medical records (EMR) of outside hospital. During the time period of the study, nearly all patients who received IVtPA at the outside hospital ERs were transferred to the hub for post IVtPA care. The standard protocol was that telestroke consult was initiated for suspected AIS patients within 8-hour window from time of last known normal. The telestroke physician would 'beam in' via highquality bidirectional audiovisual format for history and exam and with access to review neuroimaging. IVtPA cases with a negative diffusion-weighted images (DWI) were reviewed by study vascular neurologist (VHL) to confirm diagnosis. All final diagnosis was made clinically by study vascular neurologist (VHL) after reviewing clinical teams impression and diagnostic workup. If DWI showed infarct, these were automatically categorised as stroke. If DWI was negative, these were categorised as SM if an alternative diagnosis was considered clinically more likely, otherwise they were categorised as TIA. Data was collected on demographics, medical history, symptoms, door-to-needle time (DTN) initial National Institutes of Health Stroke Scale (NIHSS), functional embellishment, vital signs and neuroimaging results. Symptomatic intracerebral haemorrhage (sICH) was defined as radiographic haemorrhage accompanied by $\geq 4$ point worsening in the NIHSS. Telestroke mimic (TM) score was calculated per the described nomogram. ${ }^{1}$ Statistical analyses were performed using JMP software package (V.14). Univariate analysis was performed with $\chi^{2}$ test and Fisher's exact test as appropriate. All tests were two sided, and a $\mathrm{p}$ value was considered significant at $<0.05$.

\section{Data availlability}

Anonymised data will be shared on request from any qualified investigator.

\section{RESULTS}

Among 270 suspected AIS patients who received IVtPA via telestroke, the mean age was 65.4 (range 21-95) and 133 (49.3\%) were female. 


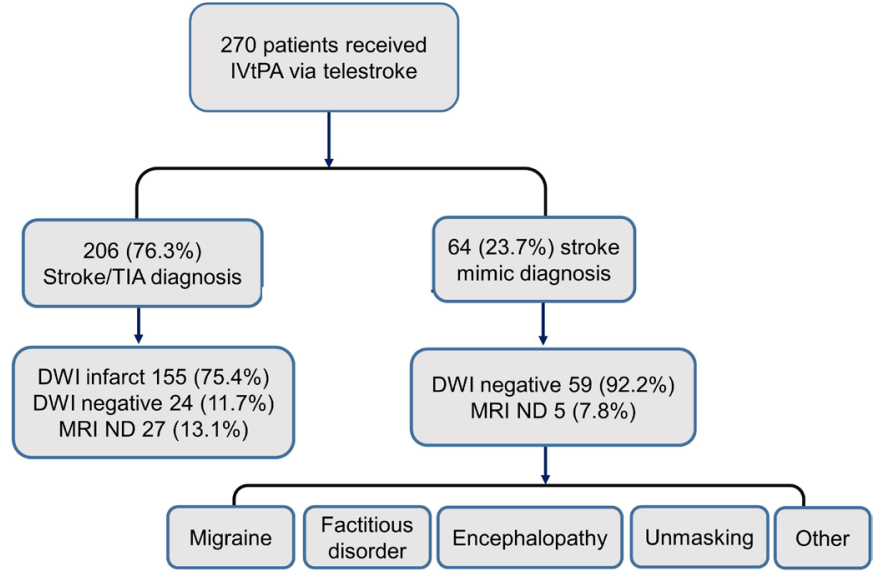

Figure 1 Patient inclusion. DWI, diffusion-weighted image; IVtPA, intravenous tissue plasminogen activator; ND, not done; TIA, transient ischemic attack.

Among 239 (88.1\%) with MRI brain DWI completed, 156 $(57.8 \%)$ were DWI+and $83(30.7 \%)$ were DWI negative. The majority $147(54.4 \%)$ of IVtPA cases were administered at the top four highest volume spoke ERs. Among 270 patients who received IVtPA for possible AIS via telestroke, we identified 64 (23.7\%) with final diagnosis of SM (figure 1). Compared with AIS patients, the SM group was younger (mean age 56.4 vs 68.2, $\mathrm{p}<0.0001$ ), more likely to be female (60.9\% vs $45.6 \%, \mathrm{p}=0.0324)$, have longer DTN times (85.3 vs $69.9 \mathrm{~min}, \mathrm{p}=0.0008)$, and longer door to tPA recommend times ( 65.0 vs $53.2 \mathrm{~min}, \mathrm{p}=0.0034$ ). SM patients did not differ from stroke patients in terms of initial NIHSS, initial systolic blood pressure or weight.

The SM diagnoses seen were migraine 26 (40.6\%), conversion $12(18.8 \%)$, encephalopathy $7(10.9 \%)$, unmasking $(9.4 \%)$ and other 13 (20.3). Other diagnoses include hyperglycaemic (3), hypoglycaemic (1), seizures (3), neoplasm (1), tongue swelling (1) spinal cord (1), syncope (1), vitreous haemorrhage (1) and peripheral nerve (1). Among the SM,
$59(92.2 \%)$ had negative DWI, and among patients without DWI, all five had negative CT brain angiogram and four had negative CT brain perfusion. No sICH was observed in the SM group. Among the SM, there were differences between the aetiologies (table 1 ).

Mean age was younger for migraine (52.0) and conversion (49.6) compared with the other subgroups $(\mathrm{p}<0.0001)$. Functional elements were noted on exam more frequently in conversion $(66.7 \%)$ and migraine $(34.6 \%)$, but rare in other diagnoses $(\mathrm{p}=0.0018)$. Among SM, the mean TM score was 14.4, (range 4.5-29.9), and only $3(4.7 \%)$ of SM had a TM score $\leq 5$. TM score $\geq 20$ was seen in $8(12.5 \%)$. Among SM, $23(35.9 \%)$ had a history of a prior similar episodes, and $15(23.4 \%)$ had a history of more than 5. Eight (12.5\%) would present with a similar recurrent episode, with two patients receiving IVtPA again in the future ( 1 conversion and 1 migraine).

\section{DISCUSSION}

Confirming stroke diagnosis on initial presentation is difficult, and prehospital scales such as Cincinnati Prehospital Stroke Scale have demonstrated good sensitivity $(84.7 \%)$ but lower positive predictive value for detecting true stroke $(56 \%)$ as opposed to $\mathrm{SM}^{2} \mathrm{SM}$ should be considered the most likely diagnosis in the majority of IVtPA-treated patients with a negative DWI on follow-up imaging, as the alternative diagnosis of IVtPA aborted infarction is relatively rare, occurring in $0.9 \%$ of patients with pretreatment positive DWI evidence of acute ischaemia. ${ }^{3} \mathrm{SM}$ often trigger acute stroke codes, and in bedside acute ER stroke code series with no time limit, SM was diagnosed in $31 \%$, with an even higher SM rate in inpatient stroke codes at $51 \% .{ }^{45}$ Large telestroke cohorts have reported a rate of SM presentations of up to $44 \% .{ }^{1}$ It is clear that SM often trigger acute stroke codes, but how often SM inadvertently go on to receive

Table 1 Stroke mimic patients

\begin{tabular}{|c|c|c|c|c|c|c|}
\hline & Migraine (26) & Conversion (12) & Encephalopathy (7) & Unmasking (6) & Other (13) & $P$ value \\
\hline Age (mean) & 52.0 & 49.6 & 79.4 & 56.3 & 59.2 & $<0.001$ \\
\hline Sex (Female) & $19(73.0 \%)$ & $7(58.3 \%)$ & $5(71.4 \%)$ & $3(50.0 \%)$ & $5(38.5 \%)$ & 0.2844 \\
\hline $\begin{array}{l}\text { Mean systolic blood } \\
\text { pressure }(\mathrm{mm} \mathrm{Hg})\end{array}$ & 143.0 & 141.9 & 160.7 & 120.5 & 168.1 & 0.0037 \\
\hline History of migraine & $11(42.3 \%)$ & $5(41.7 \%)$ & 0 & 0 & 0 & 0.0004 \\
\hline Hypertension & $17(65.4 \%)$ & $5(41.7 \%)$ & $5(71.4 \%)$ & 5 (83.3\%) & $10(76.9 \%)$ & 0.3199 \\
\hline Diabetes mellitus & $15(57.7 \%)$ & $1(8.3 \%)$ & $4(57.1 \%)$ & 2 (33.3\%) & $6(46.2 \%)$ & 0.0362 \\
\hline Functional exam & $8(30.8 \%)$ & $8(66.7 \%)$ & 0 & 0 & $1(7.8 \%)$ & 0.0006 \\
\hline
\end{tabular}

DTN, door to needle; NIHSS, National Institutes of Health Stroke Scale. 
thrombolysis is less clear. The rate of SM in thrombolysis series reported is highly variable, likely due to underreporting bias with retrospective series, which report rates as low $1.8 \%$ and as high as $14 \% .{ }^{67}$ In a GWTG (Get With The Guidelines) retrospective registry cohort, $3.5 \%$ of tPA treatments were given to SM, but as SM inclusion in the registry was optional for stroke centres, there is likely significant under-reporting bias that has to be taken into account. ${ }^{8}$ In prospective studies, rates of SM in thrombolytic cohorts are much higher. In a 5-year prospective study, $75 / 516(14.5 \%)$ of IVtPA patients were SM. ${ }^{9}$ In the NOR-TEST (The Norwegian Tenecteplase Stroke Trial 2) trial, which was a tenecteplase phase 3 trial, 181 (16.6\%) of 1091 randomised patients were SM, with migraine being the most frequent SM diagnosis. ${ }^{10}$

Saver suggested an acceptable rate of IVtPA SM at CT-based centres to be $3 \%$, but acknowledged that the calculus for risk-benefit favours the 'quickdraw' approach for IVtPA, especially given that the rate of sICH in $\mathrm{SM}$ is exceedingly low. ${ }^{11}$ However, increasing diagnostic certainty for AIS likely delays DTN times and may increase the risk of missed thrombolysis. Many thrombolysis series confirm an exceeding low rate of sICH in SM of $0 \%-0.5 \%{ }^{6-10}$ In fact, the most recent AHA guidelines in 2019 recognise this and specifically recommend thrombolysis to SM given the low rate of sICH and state that starting IVtPA is preferred over delaying treatment to pursue additional diagnostic studies. ${ }^{12}$ The acute stroke protocol for our telestroke network requires only a non-contrast CT brain for thrombolysis decision, and we do not delay thrombolysis decision to pursue advanced neuroimaging, which may explain the higher rate of SM in our thrombolysis cohort. However, the alternative of erring on the side of excluding patients with a suspected diagnosis of SM may also lead to increase overall of missed diagnosis of stroke which would increase withholding of time-sensitive treatment for otherwise eligible patients. A study of certified stroke programmes showed $22 \%$ of AIS are initially misdiagnosed with $33 \%$ of missed stroke cases presenting within 3 hours, and in $35 \%$ the diagnosis was still missed even after being seen early by neurology in the ER. ${ }^{13}$ This suggests that the risk of missing a true stroke diagnosis and withholding IVtPA is a greater concern than the risk of giving IVtPA to SM.

The TM score has been validated in external cohorts, and an operational cut-off of $\leq 5$ has been suggested as indicative of SM $(65 \%$ chance based on the score $) .{ }^{1}$ In our series, TM score $\leq 5$ was seen in only $4.7 \%$ of $\mathrm{SM}$, and therefore, its real-world usefulness appears limited. In our series, the lack of ability to see EMR of the outside hospital contributed to thrombolysis in SM, as similar prior episodes and work-up was not visible to the telestroke physician. There may also be limitations on detecting nuances of the physical exam for SM remotely.

A reasonable correlate to IVtPA clinical decision making for suspected SM is appendicitis, where clinicians err on the side of over-treatment to avoid missing true surgical cases. A negative appendectomy rate (ie, the portion of patients with suspected appendicitis clinically with normal appendices removed surgically) of $25 \%$ has been acceptable historically. ${ }^{14}$ In other clinical situations where one must balance missing a true diagnosis (and potential treatment window) versus potentially overtreating false positives, there is the acceptance of overtreatment. It has been demonstrated that improvements in the efficiency of IVtPA delivery (ie, decreasing DTN times) are associated with increased rates of SM thrombolysis rates. ${ }^{15}$ Balancing the rate of inadvertent thrombolysis of SM against the rate of undertreatment of true AIS means recognising that decreasing the former likely increases the latter. Given the differences of telemedicine compared with bedside evaluation, we suggest that compared with in-person evaluation, the proposed goal of a $3 \%$ rate of SM thrombolysis is unreasonable and centres that achieve this rate are likely undertreating or missing eligible stroke patients or delaying treatment in order to increase certainty of stroke diagnosis. In our telestroke series, $23.7 \%$ of patients who received thrombolysis were diagnosed with SM, which aligns with a high rate of SM presentations in telestroke cohorts of up to $44 \%^{1}$ and is slightly higher than a prospective bedside in person thrombolysis study showing $16.6 \%$ rate of SM. ${ }^{9} \mathrm{~A}$ higher SM thrombolysis rate for telestroke compared with the bedside rate should be acceptable, and similar to the accepted negative appendectomy rate, we suggest a reasonable goal of $25 \%$.

The strength of our study is all patients were evaluated and examined by stroke neurologists on presentation and the final diagnosis was determined by stroke neurologist. Also, as we used our telestroke database, this included all patients given IVtPA in our telestroke network, wherease since these patients would not have coded out as stroke, our GWTG registry would have not included these patients as SM reporting is optional). Limitations of our study include the fact that our telestroke platform allows only access to view imaging, but does not allow access to the all the spoke hospitals EMR. Our study shares inherent limitations associated with a retrospective review.

\section{CONCLUSION}

In our academic tertiary referral telestroke programme, $23.7 \%$ of patients administered thrombolysis had a final diagnosis of SM. We suggest that a reasonable SM thrombolysis rate for telestroke programme should be one in four, similar to the accepted negative appendectomy rate, as that the risk of overtreatment should be accepted over the risk of undertreatment.

\section{Twitter Vivien H Lee @VivienHLee}

Contributors All authors have contributed to this manuscript, including data collection, writing the manuscript, and significant editing and revision of manuscript.

Funding The authors have not declared a specific grant for this research from any funding agency in the public, commercial or not-for-profit sectors.

Competing interests None declared.

Patient consent for publication Not applicable. 
Ethics approval This study was approved by This project was approved by IRB 2017E0620. This project was approved by IRB 2017E0620. As this was a restrospective chart review with no patient contact, IRB granted us HIPAA waiver. Provenance and peer review Not commissioned; externally peer reviewed.

Open access This is an open access article distributed in accordance with the Creative Commons Attribution Non Commercial (CC BY-NC 4.0) license, which permits others to distribute, remix, adapt, build upon this work non-commercially, and license their derivative works on different terms, provided the original work is properly cited, appropriate credit is given, any changes made indicated, and the use is non-commercial. See: http://creativecommons.org/licenses/by-nc/4.0/.

ORCID iD

Vivien H Lee http://orcid.org/0000-0002-8615-198X

\section{REFERENCES}

1 Ali SF, Hubert GJ, Switzer JA, et al. Validating the TeleStroke mimic score: a prediction rule for identifying stroke mimics evaluated over TeleStroke networks. Stroke 2018;49:688-92.

2 Oostema JA, Konen J, Chassee T, et al. Clinical predictors of accurate prehospital stroke recognition. Stroke 2015;46:1513-7.

3 Freeman JW, Luby M, Merino JG, et al. Negative diffusion-weighted imaging after intravenous tissue-type plasminogen activator is rare and unlikely to indicate averted infarction. Stroke 2013;44:1629-34

4 Hand PJ, Kwan J, Lindley RI, et al. Distinguishing between stroke and mimic at the bedside: the brain attack study. Stroke 2006;37:769-75.

5 El Husseini N, Goldstein LB. "Code stroke": hospitalized versus emergency department patients. J Stroke Cerebrovasc Dis 2013;22:345-8.
6 Zinkstok SM, Engelter ST, Gensicke H, et al. Safety of thrombolysis in stroke mimics: results from a multicenter cohort study. Stroke 2013;44:1080-4.

7 Chernyshev OY, Martin-Schild S, Albright KC, et al. Safety of tPA in stroke mimics and neuroimaging-negative cerebral ischemia. Neurology 2010;74:1340-5.

8 Ali-Ahmed F, Federspiel JJ, Liang L, et al. Intravenous tissue plasminogen activator in stroke mimics findings from the get with the Guidelines-Stroke registry. Circulation: Cardiovascular Quality and Outcomes 2019;12:e005609.

9 Tsivgoulis G, Zand R, Katsanos AH, et al. Safety of intravenous thrombolysis in stroke mimics: prospective 5-year study and comprehensive meta-analysis. Stroke 2015;46:1281-7.

10 Kvistad CE, Novotny V, Næss H, et al. Safety and predictors of stroke mimics in the Norwegian tenecteplase stroke trial (NOR-TEST). Int $J$ Stroke 2019;14:508-16.

11 Saver JL, Barsan WG. Swift or sure?: the acceptable rate of neurovascular mimics among IV tPA-treated patients. Neurology 2010;74:1336-7.

12 Powers WJ, Rabinstein AA, Ackerson T. Guidelines for the early management of patients with acute ischemic stroke: 2019 update to the 2018 guidelines for the early management of acute ischemic stroke. Stroke 2019;50:e1-75.

13 Arch AE, Weisman DC, Coca S, et al. Missed ischemic stroke diagnosis in the emergency department by emergency medicine and neurology services. Stroke 2016;47:668-73.

14 Detmer DE, Nevers LE, Sikes ED. Regional results of acute appendicitis care. JAMA 1981;246:1318-20.

15 Liberman AL, Liotta EM, Caprio FZ, et al. Do efforts to decrease door-to-needle time risk increasing stroke mimic treatment rates? Neurol Clin Pract 2015;5:247-52. 